\title{
Emerging perspectives on mitochondrial dysfunction and inflammation in Alzheimer's disease
}

\author{
Seung-Min Yoo ", Jisu Park ${ }^{\#}$, Seo-Hyun Kim E Yong-Keun Jung ${ }^{*}$ \\ School of Biological Sciences, Seoul National University, Seoul 08826, Korea
}

Despite enduring diverse insults, mitochondria maintain normal functions through mitochondrial quality control. However, the failure of mitochondrial quality control resulting from excess damage and mechanical defects causes mitochondrial dysfunction, leading to various human diseases. Recent studies have reported that mitochondrial defects are found in Alzheimer's disease (AD) and worsen AD symptoms. In $\mathrm{AD}$ pathogenesis, mitochondrial dysfunction-driven generation of reactive oxygen species (ROS) and their contribution to neuronal damage has been widely studied. In contrast, studies on mitochondrial dysfunction-associated inflammatory responses have been relatively scarce. Moreover, ROS produced upon failure of mitochondrial quality control may be linked to the inflammatory response and influence the progression of $\mathrm{AD}$. Thus, this review will focus on inflammatory pathways that are associated with and initiated through defective mitochondria and will summarize recent progress on the role of mitochondria-mediated inflammation in AD. We will also discuss how reducing mitochondrial dysfunction-mediated inflammation could affect AD. [BMB Reports 2020; 53(1): 3546]

\section{INTRODUCTION}

Mitochondria play a wide range of roles in apoptosis, calcium homeostasis, cell proliferation, production of metabolic substrates, and inflammation, in addition to their primary responsibility of energy production in cells (1). Mitochondria operate various defense mechanisms from the protein level to the organelle level through mitochondrial quality control (MQC) to maintain normal functions (2). Notably, the failure of MQC results in mitochondrial dysfunction, which has been

${ }^{*}$ Corresponding author. Tel: +82-2-880-4401; Fax: +82-2-88084185; E-mail: ykjung@snu.ac.kr

${ }^{\#}$ These authors contributed equally to this work.

https://doi.org/10.5483/BMBRep.2020.53.1.274

Received 28 October 2019

Keywords: Alzheimer's disease, Dysfunction, Inflammation, Mitochondria frequently associated with many diseases (3). Interestingly, damaged mitochondria are also found in the brains of patients with $\mathrm{AD}$, and mitochondrial dysfunction is known to accelerate $A D$ symptoms. In recent years, accumulating evidence has highlighted the essential role of inflammation in AD. Here, we provide an overview of the features of inflammation and mitochondrial dysfunction, and the mechanisms underlying the mitochondria-mediated inflammatory response in $\mathrm{AD}$ pathogenesis (Fig. 1). Furthermore, we explore possible ways of adjusting MQC and inflammation to ameliorate AD symptoms and pathogenesis (Table 1).

\section{ROLE OF INFLAMMATION AND MITOCHONDRIA IN AD}

Inflammation as a central mechanism of AD

Recent findings strengthen the implication of inflammation on the pathogenesis of AD. Genetic studies have consistently identified a list of genes that can act as risk factors for $A D$, regardless of amyloid-beta $(A \beta)$ signal transduction. Triggering receptor expressed on myeloid cells 2 (TREM2) is expressed on microglial membranes, recognizes lipoproteins and phospholipids, and is involved in phagocytosis of microglial cells. Lack of TREM2 suppresses tau disease, gliosis, and neuroinflammation, because it helps the microglia respond to damage caused by tau disease (4). Besides, Apolipoprotein E (ApoE) is secreted by microglia and astrocytes and has three alleles, $\varepsilon 2, \varepsilon 3$, and $\varepsilon 4$. In the central nervous system, ApoE binds to ApoE receptors present on nerve cells to regulate the development of the central nervous system and recovery of nerve defects. Among them, the ApoE4 allele is a genetic risk factor for sporadic $A D(5)$, and as the $\varepsilon 4$ gene increases, the age of onset of $A D$ decreases. It is known that an impaired function of ApoE4 adversely affects $A \beta$ removal and $A \beta$-induced inflammatory response $(6,7)$. As mentioned above, inflammatory process-linked proteins, such as TREM2 and $A p o E$, may act independently of $A \beta$ signaling. In a bioinformatics study conducted by Zhang et al., the immuneand microglia-specific pathway, including TYROBP which is restricted to cells involved in the innate immunity, was also identified as a critical regulator of $A D$ pathogenesis (8).

Since the 1980s, researchers have found components of the immune response, such as immunoglobulins and complement

ISSN: 1976-670X (electronic edition)

Copyright (C) 2020 by the The Korean Society for Biochemistry and Molecular Biology

(c) This is an open-access article distributed under the terms of the Creative Commons Attribution Non-Commercial License (http://creativecommons.org/licenses/by-nc/4.0) which permits unrestricted non-commercial use, distribution, and reproduction in any medium, provided the original work is properly cited. 


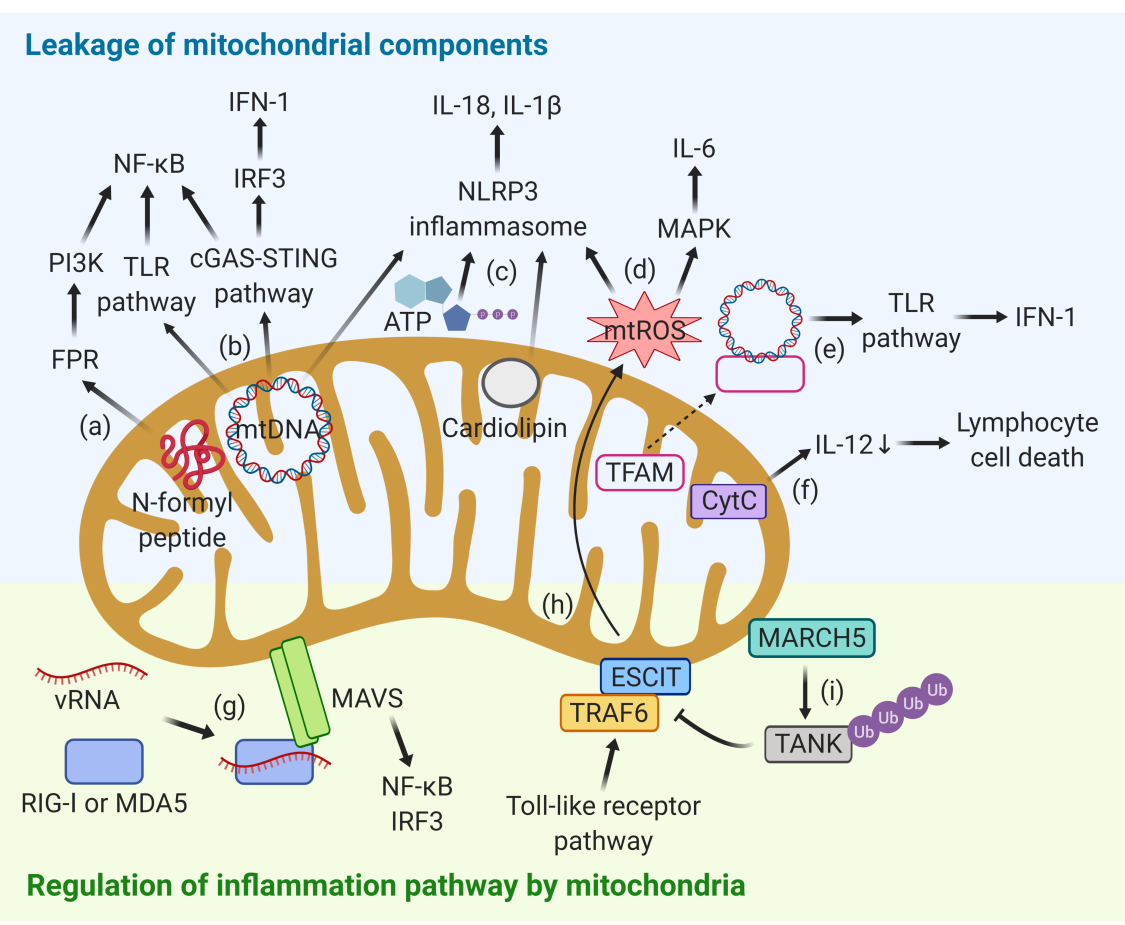

Fig. 1. Mitochondria as a regulator of inflammation. (Upper) Leakage of mitochondrial components. (a) Leaked $\mathrm{N}$-formyl peptide binds to formyl peptide receptor (FPR) to activate NF- $\mathrm{B}$. (b) Leakage of mtDNA to the cytosol activates Toll-Like-Receptor (TLR), cyclic GMP-AMP synthase-simulator of interferon genes (cGAS-STING) pathways, and NLRP3 inflammasome. (c) ATP and cardiolipin activate NLRP3 inflammasome. (d) Mitochondrial ROS (mtROS) activates NLRP3 inflammasome and mitogenactivated protein kinase (MAPK) signaling. (e) Binding of mtDNA to cytosolic TFAM activates the TLR pathway. (f) Cytosolic cytochrome $\mathrm{C}$ (Cyt C) reduces IL-12 and increases lymphocyte cell death. (Lower) Regulation of inflammation pathway by mitochondrial factors. (g) MAVS recruits RIG-I or MDA5 to viral RNA to activate NF- $\kappa B$ and IRF3. (h) ESCIT generates mtROS by binding to TRAF6. (i) MARCH5 ubiquitinates TANK to enhance TRAF6 signaling.

Table 1. Strategies to modify the stress in the mitochondria-inflammation pathway

\begin{tabular}{|c|c|c|c|c|}
\hline \multicolumn{2}{|c|}{ Targeting of mitoinflammation pathways } & \multirow{2}{*}{\begin{tabular}{l}
\multicolumn{1}{c}{ Strategies } \\
Mitochondria-targeted antioxidant \\
Mitochondrial genomic editing \\
TFAM ectopic expression
\end{tabular}} & \multirow{2}{*}{$\begin{array}{l}\text { Beneficial or detrimental* } \\
\text { Mito Q, MitoVitE, Tiron } \\
\text { mtCRISPR/Cas9, mtTALEN } \\
\text { TFAM transgenic mouse }\end{array}$} & \multirow{2}{*}{$\begin{array}{c}\text { References } \\
(112-116) \\
(42,123-125) \\
(126-129)\end{array}$} \\
\hline $\begin{array}{l}\text { Targeting mtDAMP and } \\
\text { mtPAMP }\end{array}$ & $\begin{array}{l}\text { mtROS } \\
\text { mtDNA } \\
\text { TFAM }\end{array}$ & & & \\
\hline Targeting inflammasome & NLRP3 inflammasome & $\begin{array}{l}\text { Pharmacological inhibition of } \\
\text { NLRP3 }\end{array}$ & $\begin{array}{l}\text { MCC950, JC-124, Fenamate } \\
\text { NSAIDs }\end{array}$ & $(131-133)$ \\
\hline \multirow[t]{3}{*}{$\begin{array}{l}\text { Regulating mitochondrial } \\
\text { quality control }\end{array}$} & Mitophagy & $\begin{array}{l}\text { Pharmacological enhancement of } \\
\text { mitophagy }\end{array}$ & $\begin{array}{l}\text { Nicotinamide mononucleotide } \\
(\text { NMN), Urolithin A (UA), } \\
\text { Actinonin (AC), Mitochonic acid } \\
5 \text { (MA-5) }\end{array}$ & $(136,137)$ \\
\hline & Mitochondrial dynamics & $\begin{array}{l}\text { Mitochondrial fission } \\
\text { Mitochondrial fusion }\end{array}$ & $\begin{array}{l}\text { Mdivi-1, Heptapeptide P110 } \\
{ }^{*} \text { Mfn } 2^{-/-} \text {mouse }\end{array}$ & $\begin{array}{c}(140-142) \\
(139)\end{array}$ \\
\hline & cGAS-STING pathway & Inhibition of cGAS-STING pathway & ${ }^{*} \operatorname{Prkn}{ }^{-1-}$ mouse, ${ }^{*}$ Pink $1^{-1-}$ mouse & $(145)$ \\
\hline
\end{tabular}

proteins near $A \beta$ and elevated levels of cytokines and chemokines, in $A D$ brains $(9,10)$. It has also been shown that anti-inflammatory drugs activate microglia and lower $A \beta 42$ in vivo in mouse models $(11,12)$. Several human clinical trials have revealed that anti-inflammatory drugs reduce the risk of AD $(13,14)$. Thus, many researchers now agree that an association between neuroinflammation and $A D$ pathogenesis exists and that $A D$ pathogenesis and inflammation are the cause and effect of each other, regardless of what is triggered first. In the case of acute inflammation, microglia eliminate $A \beta$ and prevent the ensuing detrimental consequences. Contrastingly, cytokines, chemokines, and ROS are over-produced by immune cells and exacerbate neurotoxicity in chronic inflammation. Whereas the former is beneficial in relieving neuropathology, the latter aggravates neurotoxicity. Next, we investigate the roles of inflammation with the opposing side to the pathogenesis of AD.

Neuroprotective inflammation in the pathogenesis of AD: Many studies have demonstrated that overexpression of inflammatory mediators in the $\mathrm{AD}$ mouse model plays a 
beneficial role in pathogenesis. Whereas aged amyloid precursor protein (APP) transgenic (TG) mice display increased production of astroglial TGF- $\beta 1$ and reduction in the number of parenchymal amyloid plaques, mice expressing hAPP and TGF- $\beta 1$ show $A \beta$ accumulation in cerebral blood vessels (15). In the study conducted by Wyss-Coray et al., researchers observed that hAPP/TGF- $\beta 1$ mice have markedly higher levels of C3, a component of the complement system, than do hAPP mice, and inhibition of $\mathrm{C} 3$ activation causes an increase in $\mathrm{A} \beta$ deposition and the number of degenerating neurons (16). Furthermore, pathogenic $A \beta$ is eliminated by immune-related clearance mechanisms. For example, low-density lipoprotein receptor-related protein 1 (LRP-1) mediates the uptake of $A \beta$ in astrocytes and neurons $(17,18)$. ATP-binding cassette subfamily A member 7 (ABCA7) also participates in phagocytic clearance of $A \beta$ in the brain (19). These results suggest that immune activation and the subsequent microglial activation help relieve $\mathrm{AD}$ pathology.

Detrimental effect of inflammation on AD: Based on various $\mathrm{AD}$ mouse models, it is known that higher levels of cytokines trigger inflammation and thereby exacerbate $A D$ pathology. The APP TG mouse model with exogenous expression of interleukin (IL)-10 in the brain displays elevated $A \beta$ accumulation and memory deficit (20). The most common mechanism whereby $A \beta$ is produced because of inflammation is inflammation-mediated regulation of APP, $\beta$-secretase 1 (BACE1), and $\gamma$-secretase expression. Activation of immunerelated transcriptional factor NK- $\mathrm{KB}$ was reported to cause APP upregulation in neurons (21). Interferon (IFN)- $\gamma$ also induces BACE1 expression through the JAK2-ERK1/2 signaling pathway in astrocytes (22). In addition, inflammation is thought to play a role in tau pathology in AD. Lipopolysaccharide (LPS) administration induces inflammation and aggravates tau pathology in the 3xTg AD mouse model (23). These results offer compelling evidence for the harmful effects of inflammation on $\mathrm{AD}$ pathogenesis.

\section{Mitochondrial cascade in AD}

Mitochondria are considered to play a critical role in the pathology of AD. Neurons need to produce large amounts of neurotransmitters and establish membrane excitability. Since mitochondria are responsible for ATP production, iron homeostasis, and $\mathrm{Ca}^{2+}$ signaling, neuronal viability relies highly on mitochondrial function. For example, mitochondria in the presynaptic nerve terminal primarily regulate presynaptic calcium at central glutamatergic terminals (24). Axon regeneration is also facilitated by increasing mitochondrial motility and recovering the energy deficit in mature neurons (25). Thus, mitochondrial defects are commonly observed in neurodegenerative diseases, including amyotrophic lateral sclerosis (ALS), Parkinson's disease (PD), and AD. In ALS and $\mathrm{PD}$, mitochondrial dysfunction and impaired mitochondrial fusion cause neuronal loss (26); mitochondria also play a pivotal role in the loss of hippocampal and cortical neurons in
AD.

Electron microscopic studies have revealed that AD brains display abnormal mitochondrial structure in the hippocampus, acoustic cortex, frontal cortex, and cerebellum (27). A recent report identified a mitochondrial fission-arrest phenotype and elongated interconnected mitochondria in the hippocampus and entorhinal cortex of patients with $\mathrm{AD}$ (28). In addition to the structural abnormality, AD brains also exhibit mitochondrial malfunction, such as changes in glucose metabolism and oxygen consumption. The activity of the pyruvate dehydrogenase complex (PDHC) and 2-ketoglutarate dehydrogenase complex is reduced in the affected regions of $A D$ brains $(29,30)$. The activity of complex I, complex II-III, and cytochrome oxidase is reduced in the cortex of AD brains (31). Increased oxidative damage to mitochondrial DNA is found in $A D$ (32). Despite these studies on the corruption of mitochondrial function in $\mathrm{AD}$ brains, it is still debatable whether mitochondrial disruption is the main cause of $A D$ or occurs as a consequence of pathological conditions in AD. This section describes the correlation between abnormal mitochondria and $\mathrm{AD}$ pathogenesis, focusing on the evidence from two conflicting hypotheses.

Mitochondrial dysfunction as an outcome of AD pathogenesis: The majority of researchers contend that $A \beta$-induced ROS generation and impaired calcium homeostasis lead to mitochondrial lesions, which are known as the secondary mitochondrial cascade. Overexpression of mutant APP in HT22 mouse hippocampal cell line results in defective mitochondrial dynamics and changes mitochondrial structure and function in neurons (33). APP can accumulate in mitochondrial import channels of $\mathrm{AD}$ brains and cause mitochondrial dysfunction (34). It has also been reported that $A \beta$ directly disrupts mitochondrial function and inhibits key enzymatic activities. Lustbader et al. reported that alcohol dehydrogenase (ABAD) interacts with $A \beta$ and mediates $A \beta$-induced apoptosis and free-radical generation in neurons (35). AD brains express higher levels of voltage-dependent anion-selective channel 1 (VDAC1), which interacts with $A \beta$ and phosphorylated tau to block mitochondrial pores, precipitating mitochondrial dysfunction (36).

In addition, there are reports showing that mitochondrial fusion and fission factors are affected by $A \beta$. A $\beta$ induces oxidative stress that triggers mitochondrial fragmentation through decreased mitofusin-2 (Mfn2) expression by activating cyclin-dependent kinase 5 (Cdk5)-mediated peroxidase 2 (Prx2) phosphorylation (37). A $\beta$ also mediates dynamin-related protein 1 (Drp1) phosphorylation via AKT activation, promoting excessive mitochondrial fission and leading to neuronal apoptosis (38). Collectively, the results suggest that the accumulation of mitochondrial APP and A $\beta$ contributes to the defective energy metabolism and mitochondrial abnormalities seen in AD.

Mitochondrial defects as a causative factor of AD: In stark contrast to the above, several studies have also implied that 
mitochondrial failure drives disease progression, which is known as the primary mitochondrial hypothesis. Mitochondrial loss leads to changes in ROS generation, altered calcium homeostasis, failure of mitochondrial homeostasis, and cell death. Neuron-specific deficiency of cytochrome $C$ oxidase (COX) leads to a decrease in amyloid plaques, A $\beta 42$ levels, $\beta$-secretase activity, and oxidative damage in the mouse model expressing mutant APP and PS1 (39). Other groups also claimed that high levels of mitochondrial DNA (mtDNA) deletion could cause COX deficiency (40). In the same context, an ultrasensitive next-generation sequencing analysis revealed an increase in mtDNA mutation frequency in $A D$ brains $(41,42)$. Furthermore, injection of the mitochondrial complex I inhibitor rotenone into rats triggers tauopathy in the striatum (43). Whereas molecular mechanisms that corroborate the secondary mitochondrial hypothesis have been identified, there is only phenomenological evidence for the primary mitochondrial hypothesis that mitochondrial impairments dictate AD pathology. Nonetheless, based on the above observations, mitochondrial failure possibly facilitates $\mathrm{AD}$ pathogenesis.

\section{RELATIONSHIP BETWEEN MITOCHONDRIA AND INFLAMMATION}

\section{Mitochondrial quality control}

Because mitochondria in eukaryotic cells are the major organelles that provide ATP through the electron-transport chain (ETC) and ETC inevitably generates ROS, mitochondrial DNA, proteins, and lipids are damaged first, causing mitochondrial dysfunction (44). As mentioned earlier, however, MQC preserves the normal function of mitochondria. At the molecular level, mitochondria have a specific DNA polymerase subunit gamma (PolG) for mtDNA repair (45) and chaperones, such as Hsp60/70, to repair misfolded proteins (46). Mitochondrial AAA protease in the mitochondrial intermembrane space, Chip in the mitochondrial inner membrane, and LON protease in the mitochondrial matrix decompose damaged and misfolded proteins (47). Damaged proteins in the mitochondrial envelope are also ubiquitinated by the E3 ubiquitin-protein ligase MARCH5 and degraded by proteasomes (48). Antioxidants can directly quench ROS to prevent ROS-mediated damages from occurring (45). At the organelle level, mitochondrial biogenesis responds to a variety of stress conditions, such as calorie restriction, exercise, NO, CO, and ROS. This process creates new mitochondria (49) and promotes mitochondrial fusion through MFN and optic atrophy 1 (OPA1) to compensate for the deficient components. Damaged mitochondria are separated by the fission process including Drp1 or by the budding from the mitochondrial membrane. Damaged mitochondria are wrapped in autophagosomes and then eventually degraded within lysosomes (2).

\section{Mitochondria in inflammation}

Inflammation involves pathogen-associated molecular patterns (PAMPs) presented by pathogens or external ligands and damage-associated molecular patterns (DAMPs), which are endogenous molecules released into the extracellular space because of tissue damage. PAMPs and DAMPs are recognized by pattern recognition receptors (PRRs), which generate innate immunity-related substances through intracellular signaling pathways. Mitochondria have many similarities to bacteria, so the escape of mitochondrial content into the cytosol or the extracellular space serves as a PAMP or DAMP, invoking PRR signaling (50). Hence, mitochondria act as regulators of inflammatory signaling.

\section{Inflammation triggered by the leakage of mitochondrial components}

Mitochondrial DNA: Mitochondria originated from $\alpha$-proteobacteria through endosymbiosis in ancient eukaryotes (51) and have circular DNA and CpG motifs similar to those of bacteria (52). Circular DNA with unmethylated CpG motifs interacts with Toll-like receptor 9 (TLR9) and activates NF-אB (53). In addition, oxidized mtDNA binds to the leucine-rich repeat pyrin domain containing 3 (NLRP3) to activate the NLRP3 inflammasome and increase interleukin-1 beta (IL-1 $\beta$ ) during cell death (54). The cyclic GMP-AMP synthase (cGAS) stimulator of interferon genes (STING) pathway also recognizes mtDNA in the cytosol and produces IFN-1 through the interferon regulatory factor 3 (IRF3) and NF- $\mathrm{\kappa B}(55)$.

$\mathrm{N}$-formyl peptides: Mitochondria synthesize bacteria-like peptides using 22 tRNAs and 2 mitochondrial ribosomes, and like those of prokaryotes, mitochondrial proteins are characterized by the presence of formyl-methionine (formyl-Met) at the N-terminal (56). The mitochondrial formyl-Met acts as a chemoattractant for neutrophils when exposed outside of the cells (57) and formyl-peptides bind to the formyl peptide receptors (FPRs) to activate the FPR signaling pathway in neutrophils (58). In experiments with formyl methionineleucine-phenylalanine ( $\mathrm{fMLP}$ ), $\mathrm{fMLP}$ induces IL-1 $\beta$ production through a rapid increase in PI3K- NF-KB activity (59).

Cardiolipin: Cardiolipin is a phospholipid that constitutes about $20 \%$ of the mitochondrial inner membrane. This phospholipid is common in bacteria but is found only in the mitochondrial inner membrane in eukaryotic cells. Cardiolipin regulates mitochondrial dynamics, apoptotic signaling, mitophagy, and ROS generation by noncovalent interactions (60). Exposure of cardiolipin on the mitochondrial outer membrane activates NLRP3 inflammasome by directly binding to NLRP3, resulting in the production of IL-1 $\beta$ (61). Externalized cardiolipin also induces mitophagy by binding to light-chain 3 (LC3), a receptor for autophagy (62).

ATP: Under normal conditions, extracellular ATP is rapidly degraded by the nucleotidases CD39 and CD73 (63). However, an acute increase in extracellular ATP induces IL-18 by binding to P2X7 receptor and activating NLRP3 inflammasome 
in macrophages (64). In the acute lung-injury model, LPS-mediated inflammation leads to a temporary accumulation of ATP in the airways of mice. Treatment with apyrase to degrade extracellular ATP reduces LPS-mediated inflammation (65).

Cytochrome C: Cytochrome C (Cyt C) is a protein that transports electrons from complex III to complex IV of ETC in the mitochondrial inner membrane. The release of Cyt $\mathrm{C}$ into the cytosol induces apoptosis by binding to Apaf- 1 , but the role of extracellular Cyt $C$ is not clear. Some reports have shown that Cyt $C$ may affect inflammation (66). Cyt $C$ injected into $\mathrm{CD}^{+}$dendritic cells reduces IL-12 production (67). Extracellular Cyt $\mathrm{C}$ promotes lymphocyte death and leucine-rich alpha-2-glycoprotein-1 (LRG1) binds to Cyt C and reduces its toxicity (68). Additionally, it has been shown that extracellular Cyt $\mathrm{C}$ increases NF- $\mathrm{\kappa B}$ activity and cytokine production in mouse spleen cells (69).

Mitochondrial ROS: In general, ROS induces functional errors by oxidizing proteins, lipids, and DNA. Mitochondria produce energy through ETC, which also generates mitochondrial ROS (mtROS) during electron transfer at the complex I and III in the mitochondrial inner membrane (70). The mtROS is released into the cytoplasm when high concentrations of $\mathrm{Ca}^{2+}$ and cyclophilin D convert the ATP synthase into a non-specific pore or when ROS opens mitochondrial permeability transition pores (71) and drives proinflammatory cytokine production. Inhibition of mtROS reduces IL-6 levels produced by LPS treatment (72), whereas mtROS increases IL-1 $\beta$ by activating NLRP3 inflammasomes and induces IL-6 production through inflammasome-independent transcriptional regulation (73).

Mitochondrial transcription factor A: The primary role of mitochondrial transcription factor $A$ (TFAM) is to regulate nucleoids, a condensed form of mitochondrial DNA. TFAM deficiency induces mtDNA mutations and mtDNA escapes into the cytosol, where it induces Type I IFN production through the cGAS-STING pathway (74). Additionally, extracellular TFAM is inactive, but has structural homology with HMGB1, which binds to DNA and induces inflammation (75). Similarly, binding of TFAM to mtDNA activates Type 1 IFN in plasmacytoid dendritic cells through the RAGE-TLR9 signaling pathway (76).

\section{Regulators of inflammatory signaling pathway in mitochondria}

Mitochondrial antiviral signaling protein: Retinoic acid-inducible gene 1 receptor (RIG1)-like receptors RIG-I and MDA5 recognize different types of viral RNA (vRNA) in the cytosol and bind to mitochondrial antiviral signaling protein (MAVS) on the mitochondrial outer membrane or peroxisome by interacting via caspase activation and recruitment domains (CARDs) (77). MAVS present on the mitochondrial outer membrane activates NF-kB and IRF3 (78) or recruits NLRP3 inflammasomes to mitochondria for IL-1 $\beta$ production (79). In addition, MAVS is activated by mitochondrial dynamics; mitochondrial elongation induces MAVS activation, but its fission decreases MAVS expression (80). MFN2, which is required for mitochondrial fusion, directly binds to MAVS and inhibits its activity (81). Mitochondrial dynamics affect MAVS, probably because of the need for self-oligomerization for activation (82).

Evolutionarily conserved signaling intermediate in Toll pathway: Evolutionarily conserved signaling intermediate in Toll pathway (ECSIT) was identified as a TRAF- 6 binding protein and is an E3 ligase involved in the TLR signaling pathway. ECSIT is a cytosolic protein, but interacts with the chaperone NDUFAF1 and traffics to the mitochondria to regulate complex I assembly (83). In the TLR signaling pathway, ECSIT binds to TRAF6 to recruit mitochondria to the phagosomal membrane and produce mtROS (84). An increase in constitutive mtROS production in ECSIT-deleted macrophages prevents further TLR-induced mtROS production, demonstrating the key role of ECSIT in mtROS production and mitophagydependent MQC (85).

Membrane-associated ring finger (C3HC4) 5: Membraneassociated ring finger (C3HC4) $5(\mathrm{MARCH} 5)$, an E3 ligase present in the mitochondrial outer membrane, increases inflammation by poly-ubiquitinating and attenuating TANK, a TRAF-interacting protein. TANK inhibits TRAF6 in the TLR7 signaling pathway, revealing a role of mitochondria in modulating innate immunity and linking mitochondria to the TLR signaling pathway (86).

\section{MITOCHONDRIAL-INFLAMMATION AXIS IN AD PATHOLOGY}

\section{Evidence for mitochondrial DAMPs and PAMPs in AD pathology}

As discussed, mitochondrial DAMPs and PAMPs can activate inflammation. In the central nervous system (CNS), they initiate pro-inflammatory immune responses in glial cells, thereby leading to chronic neuroinflammation and accelerating the pathology of neurodegenerative diseases, including $A D$ (87-89).

Mitochondrial DNA: It has been shown that mtDNA induces neuroinflammation in vivo. Injection of mitochondrial lysates or mtDNA into the hippocampal dentate gyri triggers pro-inflammatory signaling (90). Hippocampal injection of mitochondria or mtDNA leads to NF- $\kappa B$ phosphorylation, induction of TNF $\alpha$ mRNA, and a decrease in TREM2 expression, all of which are closely associated with AD pathology (91-93) and are involved in anti-inflammatory and phagocytic pathways $(94,95)$. Simultaneously, hippocampal injection of mtDNA increases astrocyte proliferation with elevated levels of cortical colony-stimulating factor 1 receptor (CSF1R) and GFAP proteins. Interestingly, mitochondrial lysates also upregulate endogenous APP and A $\beta$ (90), strongly supporting the correlation between mtDNA and AD pathology. Moreover, 
the relevance of mtDNA levels to AD pathology was reported (96). Circulating cell-free mtDNA is profoundly downregulated in the cerebrospinal fluid (CSF) of patients with sporadic AD as well as asymptomatic subjects at risk (96). Notably, preclinical subjects with pathogenic mutations in PSEN1 exhibit a reduction in the mtDNA concentration in CSF before other AD-related biomarkers in CSF can be detected, highlighting the use of mtDNA as a potential AD biomarker. A recent study using post-mortem brain tissues reported the regional differences in mtDNA levels in human brains; the mtDNA levels in the parietal cortex is lower in non-diabetic $A D$ subjects, but not in diabetic AD patients than in non-cognitively impaired controls (97).

Cardiolipin: It has been described that aged brains, in addition to elevated ROS production, have lower levels of cardiolipin $(98,99)$. In contrast, the proportion of peroxidized cardiolipin is higher in the brains of aged rodents (100), which results in various mitochondrial defects, such as low respiratory chain efficiency and elevated ROS production (99-101). This excessive level of ROS may contribute to chronic inflammation. Activation of the NLRP3 inflammasome is attenuated by pharmacological inhibitors of ROS production (61). Therefore, age-related cardiolipin oxidation results in mitochondrial dysfunction and aberrant ROS production that subsequently provokes chronic inflammation.

Mitochondrial transcription factor A: TFAM is implicated in the inflammation of the CNS in neurodegenerative diseases (89). TFAM upregulates secretion of IL-6 and cytotoxins in primary microglia that were obtained from post-mortem human samples or THP-1 human monocytic cells, a model of human microglia (102). Administration of TFAM into the cisterna magna in the rodent model increases levels of pro-inflammatory cytokines, including IL- $1 \beta$, IL- 6 , and TNF- $\alpha$, in the hippocampus and frontal cortex (103), which are the predominant regions affected by $\mathrm{AD}$ (89). These results underscore the ability of extracellular TFAM to induce pro-inflammatory responses in microglia. Furthermore, when combined with CpG-rich mtDNA, TFAM can activate RAGE to mediate a pro-inflammatory immune response and promote the production of TNF- $\alpha$ via the PI3K/AKT and ERK pathways (104). Accordingly, blocking RAGE with antagonistic antibodies inhibits the secretion of monocyte chemotactic protein-1 (MCP-1) in TFAM-stimulated THP-1 cells (103). Considering that RAGE binds to $A \beta(105,106)$ and that microglia express high levels of RAGE in patients with AD (107), the TFAM-RAGE pathway may potentially play a role in $A D$ pathogenesis (87).

Cytochrome C: Given that Cyt C is critical for the regulation of apoptosis, it has been implicated in the excessive cell death observed during the progression of $\mathrm{AD}$. Reports showed that CSF Cyt C levels are increased in patients with $\mathrm{MCl}(87,108)$. Whereas the release of Cyt $C$ from mitochondria is considered to be a mediator of cell death in AD (109), Cyt C released into the extracellular space may be able to provoke PAMP responses (87). Mouse splenocytes exposed to Cyt C show pro-inflammatory activity, including the release of inflammatory mediators such as chemokine ligand 5 (CCL5)/RANTES, CCL3/MIP-1 $\alpha$, and MCP-1 (69). As circulating Cyt C is increased in many chronic inflammatory diseases, such as liver injury, SIRS, and myocardial infarction (110), extracellular Cyt $\mathrm{C}$ could also activate microglia-like cells to exacerbate inflammatory damage, probably by interacting with TLR4 on microglia (111).

\section{STRATEGIES FOR TARGETING MITOINFLAMMATION PATHWAYS}

\section{Targeting mtDAMP and mtPAMP}

Mitochondrial ROS: Oxidative stress mediates mitochondrial damages during aging, particularly by damaging mtDNA and peroxidizing cardiolipin, and generates excessive ROS in turn as a byproduct. In this regard, the mitochondria-targeted antioxidants (MTAs) that specifically curtail oxidative stress within mitochondria have greater advantages than do untargeted cellular antioxidants (112). MTAs can cross the mitochondrial phospholipid bilayer and sequester ROS where it is generated. MTAs, such as MitoQ and MitoVitE, are more efficient in alleviating the damage caused by excessive ROS levels and blocking apoptosis than are untargeted antioxidants (113). Particularly, MitoQ is protective in the aged rodent model of neurodegenerative diseases, such as PD $(114,115)$, and suppresses the NLRP3 inflammasome-mediated production of inflammatory cytokines in THP-1 cells (116). In addition, the release of metals can further exacerbate the oxidative damage mediated by high ROS levels (112). Tiron, one of the MTAs, can confer marked protection against mtROS, because it targets not just ROS but also free intracellular metals that are released as a consequence of oxidative stress (112). Since iron accumulation and mtROS synergistically contribute to neurodegenerative pathology in $\mathrm{AD}$ and PD $(117,118)$, and chronic inflammation in microglia is characterized by an increase in intracellular iron levels (119), Tiron may mitigate chronic inflammation by reducing iron-mediated ROS stress in neurodegenerative disorders.

Mitochondrial DNA: mtDNA is relatively unstable and vulnerable to oxidative insults because they lack histones and have a limited enzymatic repair system. As a result, mtDNA mutations accumulate during aging $(120,121)$ and are a significant risk factor for $\operatorname{AD}(41,122)$. Mutated mtDNA can be revised via genome-editing technologies, such as clustered regularly interspaced short palindromic repeats/associated protein 9 (CRISPR/Cas9) and transcription activator-like effector nucleases (TALENs) $(42,123)$. This strategy to revise the mutated mtDNA involves expressing a gRNA targeting the pathogenic mtDNA and mitoCas9 that is localized to the mitochondrial matrix and specifically cleaves the mtDNA. In addition, mitoTALENs were used to eliminate pathogenic mtDNA and thus recover respiratory capacity and improve 
oxidative phosphorylation (124).

Mitochondrial transcription factor A: Overexpression of mitochondrial TFAM exerted beneficial effects in model systems for aging-related hearing loss (125), memory loss (126), and AD $(127,128)$. In TFAM TG mice, age-related symptoms, such as mitochondrial deficits in the brain, motor learning memory, working memory, and hippocampal long-term potentiation (LTP), are alleviated (126). Remarkably, IL-1 $\beta$ was significantly reduced in aged TFAM TG mice, indicating compensatory suppression of the TFAM-mediated aberrant inflammatory response. TFAM overexpression also exhibits a protective effect in the 3xTg AD mouse model (PS1M146V, APPswe, and MAPT P301L triple TG), reducing cognitive dysfunction, mtDNA oxidative stress, and A $\beta$ accumulation (128).

\section{Targeting the inflammasome}

Byproducts of mitochondrial dysfunction, such as mtROS and mtPAMP, can regulate the pro-inflammatory response by activating the inflammasome (123). Using Nlrp3 knockout and Caspase-1 knockout mice, the NLRP3/caspase-1 axis was shown to play an important role in the pathogenesis of $A D$ (129). In agreement, inhibitors of the NLRP3 inflammasome ameliorate $A D$ pathology in animal models of AD (130-132). MCC950, which inhibits inflammasome and microglial activation in the APP/PS1 mouse model of AD (131), might inhibit NLRP3-induced oligomerization of ASC, a key adaptor protein that is required for the activation of the inflammasome (133). In addition, several clinically approved fenamate NSAIDs inhibit the NLRP3 inflammasome via the blockade of the volume-regulated anion channels (VRAC), a $\mathrm{Cl}$ channel, and consequently ameliorate cognitive impairment in animal models of AD (130).

\section{Regulating mitochondrial quality control}

Mitophagy: Tight regulation of MQC by facilitating mitophagy and subsequent inhibition of chronic inflammation were suggested as a potential therapeutic strategy for AD (134). A recent study by Fang et al. showed that enhancing mitophagy prevents $A D$ pathology, including cognitive impairment, tau hyper-phosphorylation, $A \beta$ accumulation, and neuroinflammation (135), highlighting the importance of MQC in AD intervention. Furthermore, mitochonic acid 5 (MA-5) was shown to regulate mitophagy via BCL2/adenovirus E1B 19-kDa protein-interacting protein 3 (BNIP3), reducing mitochondrial apoptosis in BV-2 cells (136). Mitophagy may inhibit inflammation by downregulating ROS-producing mitochondria, since blocking mitophagy results in the increase of ROS, followed by NLRP3 activation (137).

Mitochondrial dynamics: Several studies reported that an imbalance of mitochondrial dynamics induces chronic inflammatory stress and thus aggravates the pathogenesis of neurodegenerative disorders. Disruption of mitochondrial fusion by Mfn2 knockout in the hippocampus results in excessive mitochondrial fragmentation and inflammatory response, which are the characteristic features of $\mathrm{AD}$ pathology (138). In contrast, negative regulation of mitochondrial fission by genetic or pharmacological methods significantly alleviates inflammation. Inhibiting mitochondrial fission by Mdivi-1, a chemical inhibitor of Drp1 or Drp1 knockdown, reduces pro-inflammatory signaling in the LPS-stimulated BV-2 cells (139) and a kainic acid-injected rodent model (140). Recently, Joshi et al. demonstrated that neurotoxicity can be directly attributed to the release of neurotoxic proteins from microglia displaying Drp1 and Fis1-mediated mitochondrial fragmentation, followed by the activation of naive astrocytes to the A1 state (141). This neurotoxicity could be reversed by the treatment with a heptapeptide (P110) that blocks the Drp1-Fis1 interaction. Interestingly, AD patients show a distinct pattern of mitochondrial dynamics (142). AD mitochondria exhibit significant fragmentation in a Drp1-dependent manner, whereas $\mathrm{MCl}$ mitochondria have increased mitochondrial Mfn2 levels, likely promoting mitochondrial fusion. These changes in mitochondrial dynamics may contribute to the induction of pro-inflammatory signaling in microglial cells. Taken together, subtle regulation of mitochondrial dynamics during disease progression may be a possible therapeutic strategy to relieve inflammatory stress and thus alleviate $A D$ pathology.

\section{cGAS-STING pathway}

Binding of oxidized mtDNA to cGAS results in the translocation of STING to the Golgi apparatus, leading to phosphorylation of the transcription factor IRF3 and activation of NF-KB signaling (143). The cGAS-STING pathway has also been found to be involved in autophagy in innate immune cells (55). Activation of the cGAS-STING pathway promotes mitophagy through cGAS/beclin-1 interaction, which in turn negatively regulates cGAS activity and increases cytosolic DNA degradation (144). A recent study has elucidated that aberrant mitophagy in Prkn or Pink1 knockout mice leads to a strong inflammatory phenotype, which is mitigated by genetic inactivation of STING (145). Thus, the cGAS-STING pathway may be a potent therapeutic target to counter mitoinflammation.

\section{CONCLUSION}

Mitochondrial functions and inflammatory signals are closely linked to AD symptoms and pathogenesis. In this review, we described mitochondrial components as being causative factors of inflammation, but simultaneously are suitable therapeutic targets in regulating the neuroinflammation (Fig. 1, Table 1). Indeed, inhibiting mitochondrial inflammation or maintaining functional mitochondria through MQC reverts many symptoms observed in the $\mathrm{AD}$ model. Thus, mitochondrial inflammation is a valuable diagnostic target and requires further study as an emerging therapeutic target for treating AD. 


\section{ACKNOWLEDGEMENTS}

This work was supported by a Bio \& Medical Technology Development Program of the National Research Foundation (NRF-2017M3A9G7073521) and a CRI grant (NRF-2019R1A 2B5B03070352) funded by the Ministry of Education, Science and Technology, Korea.

\section{CONFLICTS OF INTEREST}

The authors have no conflicting interests.

\section{REFERENCES}

1. Smith RA, Hartley RC, Cocheme HM and Murphy MP (2012) Mitochondrial pharmacology. Trends Pharmacol Sci 33, 341-352

2. Yoo SM and Jung YK (2018) A Molecular Approach to Mitophagy and Mitochondrial Dynamics. Mol Cells 41, 18-26

3. Suomalainen A and Battersby BJ (2018) Mitochondrial diseases: the contribution of organelle stress responses to pathology. Nat Rev Mol Cell Biol 19, 77-92

4. Leyns CEG, Ulrich JD, Finn MB et al (2017) TREM2 deficiency attenuates neuroinflammation and protects against neurodegeneration in a mouse model of tauopathy. Proc Natl Acad Sci U S A 114, 11524-11529

5. Corder EH, Saunders AM, Strittmatter WJ et al (1993) Gene dose of apolipoprotein $\mathrm{E}$ type 4 allele and the risk of Alzheimer's disease in late onset families. Science 261, 921-923

6. Robert J, Button EB, Yuen B et al (2017) Clearance of beta-amyloid is facilitated by apolipoprotein $\mathrm{E}$ and circulating high-density lipoproteins in bioengineered human vessels. Elife 6, e29595.

7. Tai LM, Ghura S, Koster KP et al (2015) APOE-modulated Abeta-induced neuroinflammation in Alzheimer's disease: current landscape, novel data, and future perspective. J Neurochem 133, 465-488

8. Zhang B, Gaiteri C, Bodea LG et al (2013) Integrated Systems Approach Identifies Genetic Nodes and Networks in Late-Onset Alzheimer's Disease. Cell 153, 707-720

9. Griffin WS, Stanley LC, Ling C et al (1989) Brain interleukin 1 and S-100 immunoreactivity are elevated in Down syndrome and Alzheimer disease. Proc Natl Acad Sci U S A 86, 7611-7615

10. Eikelenboom $P$ and Stam FC (1982) Immunoglobulins and complement factors in senile plaques. An immunoperoxidase study. Acta Neuropathol 57, 239-242

11. Eriksen JL, Sagi SA, Smith TE et al (2003) NSAIDs and enantiomers of flurbiprofen target $\gamma$-secretase and lower A $\beta 42$ in vivo. J Clin Invest 112, 440-449

12. Yan Q, Zhang J, Liu H et al (2003) Anti-Inflammatory Drug Therapy Alters $\beta$-Amyloid Processing and Deposition in an Animal Model of Alzheimer's Disease. J Neurosci 23, 7504-7509

13. Zandi PP, Anthony JC, Hayden KM, Mehta K, Mayer L and Breitner JCS (2002) Reduced incidence of AD with NSAID but not not $\mathrm{H} 2$ receptor antagonists: the Cache County Study. Neurology 59, 880-886

14. Breitner JC, Welsh KA, Helms MJ et al (1995) Delayed onset of Alzheimer's disease with nonsteroidal antiinflammatory and histamine $\mathrm{H} 2$ blocking drugs. Neurobiol Aging 16, 523-530

15. Wyss-Coray T, Lin C, Yan F et al (2001) TGF-beta1 promotes microglial amyloid-beta clearance and reduces plaque burden in transgenic mice. Nat Med 7, 612-618

16. Wyss-Coray T, Yan F, Lin AHT et al (2002) Prominent neurodegeneration and increased plaque formation in complement-inhibited Alzheimer's mice. Proc Natl Acad Sci U S A 99, 10837-10842

17. Liu CC, Hu J, Zhao N et al (2017) Astrocytic LRP1 Mediates Brain Abeta Clearance and Impacts Amyloid Deposition. J Neurosci 37, 4023-4031

18. Kanekiyo T, Cirrito JR, Liu CC et al (2013) Neuronal clearance of amyloid-beta by endocytic receptor LRP1. J Neurosci 33, 19276-19283

19. Fu Y, Hsiao JH, Paxinos G, Halliday GM and Kim WS (2016) ABCA7 Mediates Phagocytic Clearance of Amyloid-beta in the Brain. J Alzheimers Dis 54, 569-584

20. Chakrabarty P, Li A, Ceballos-Diaz C et al (2015) IL-10 alters immunoproteostasis in APP mice, increasing plaque burden and worsening cognitive behavior. Neuron 85, 519-533

21. Grilli $M$, Ribola $M$, Alberici A, Valerio A, Memo $M$ and Spano P (1995) Identification and characterization of a kappa $\mathrm{B} /$ Rel binding site in the regulatory region of the amyloid precursor protein gene. J Biol Chem 270, 26774-26777

22. Cho HJ, Kim SK, Jin SM et al (2007) IFN-gamma-induced BACE1 expression is mediated by activation of JAK2 and ERK1/2 signaling pathways and direct binding of STAT1 to BACE1 promoter in astrocytes. Glia 55, 253-262

23. Sy M, Kitazawa $M$, Medeiros R et al (2011) Inflammation induced by infection potentiates tau pathological features in transgenic mice. Am J Pathol 178, 2811-2822

24. Billups B and Forsythe ID (2002) Presynaptic Mitochondrial Calcium Sequestration Influences Transmission at Mammalian Central Synapses. J Neurosci 22, 5840-5847

25. Zhou B, Yu P, Lin $M-Y$, Sun $T$, Chen $Y$ and Sheng $Z H$ (2016) Facilitation of axon regeneration by enhancing mitochondrial transport and rescuing energy deficits. J Cell Biol 214, 103-119

26. Tang FL, Liu W, Hu JX et al (2015) VPS35 Deficiency or Mutation Causes Dopaminergic Neuronal Loss by Impairing Mitochondrial Fusion and Function. Cell Rep $12,1631-1643$

27. Johnson $A B$ and Blum NR (1970) Nucleoside phosphatase activities associated with the tangles and plaques of alzheimer's disease: a histochemical study of natural and experimental neurofibrillary tangles. J Neuropathol Exp Neurol 29, 463-478

28. Zhang L, Trushin S, Christensen TA et al (2016) Altered brain energetics induces mitochondrial fission arrest in Alzheimer's Disease. Sci Rep 6, 18725

29. Gibson GE, Sheu KF, Blass JP et al (1988) Reduced activities of thiamine-dependent enzymes in the brains 
and peripheral tissues of patients with Alzheimer's disease. Arch Neurol 45, 836-840

30. Sorbi S, Bird ED and Blass JP (1983) Decreased pyruvate dehydrogenase complex activity in Huntington and Alzheimer brain. Ann Neurol 13, 72-78

31. Mutisya EM, Bowling AC and Beal MF (1994) Cortical Cytochrome Oxidase Activity Is Reduced in Alzheimer's Disease. J Neurochem 63, 2179-2184

32. Mecocci P, MacGarvey U and Beal MF (1994) Oxidative damage to mitochondrial DNA is increased in Alzheimer's disease. Ann Neurol 36, 747-751

33. Reddy PH, Yin X, Manczak M et al (2018) Mutant APP and amyloid beta-induced defective autophagy, mitophagy, mitochondrial structural and functional changes and synaptic damage in hippocampal neurons from Alzheimer's disease. Hum Mol Genet 27, 2502-2516

34. Devi L, Prabhu BM, Galati DF, Avadhani NG and Anandatheerthavarada HK (2006) Accumulation of Amyloid Precursor Protein in the Mitochondrial Import Channels of Human Alzheimer's Disease Brain Is Associated with Mitochondrial Dysfunction. J Neurosci 26, 9057-9068

35. Lustbader JW, Cirilli M, Lin C et al (2004) ABAD Directly Links $A \beta$ to Mitochondrial Toxicity in Alzheimer's Disease. Science 304, 448-452

36. Manczak M and Reddy PH (2012) Abnormal interaction of VDAC1 with amyloid beta and phosphorylated tau causes mitochondrial dysfunction in Alzheimer's disease. Hum Mol Genet 21, 5131-5146

37. Park J, Choi H, Min JS et al (2015) Loss of mitofusin 2 links beta-amyloid-mediated mitochondrial fragmentation and Cdk5-induced oxidative stress in neuron cells. J Neurochem 132, 687-702

38. Kim DI, Lee KH, Gabr AA et al (2016) A $\beta$-Induced Drp1 phosphorylation through Akt activation promotes excessive mitochondrial fission leading to neuronal apoptosis. Biochim Biophys Acta 1863, 2820-2834

39. Fukui H, Diaz F, Garcia S and Moraes CT (2007) Cytochrome c oxidase deficiency in neurons decreases both oxidative stress and amyloid formation in a mouse model of Alzheimer's disease. Proc Natl Acad Sci U S A 104, 14163-14168

40. Krishnan KJ, Ratnaike TE, De Gruyter HLM, Jaros E and Turnbull DM (2012) Mitochondrial DNA deletions cause the biochemical defect observed in Alzheimer's disease. Neurobiol Aging 33, 2210-2214

41. Hoekstra JG, Hipp MJ, Montine TJ and Kennedy SR (2016) Mitochondrial DNA mutations increase in early stage Alzheimer disease and are inconsistent with oxidative damage. Ann Neurol 80, 301-306

42. Coskun PE, Beal MF and Wallace DC (2004) Alzheimer's brains harbor somatic mtDNA control-region mutations that suppress mitochondrial transcription and replication. Proc Natl Acad Sci U S A 101, 10726-10731

43. Höglinger GU, Lannuzel A, Khondiker ME et al (2005) The mitochondrial complex I inhibitor rotenone triggers a cerebral tauopathy. J Neurochem 95, 930-939

44. Lopez-Otin C, Blasco MA, Partridge L, Serrano M and Kroemer G (2013) The hallmarks of aging. Cell 153, 1194-1217
45. Scheibye-Knudsen M, Fang EF, Croteau DL, Wilson DM and Bohr VA (2015) Protecting the mitochondrial powerhouse. Trends Cell Biol 25, 158-170

46. Hammerling BC and Gustafsson AB (2014) Mitochondrial quality control in the myocardium: Cooperation between protein degradation and mitophagy. J Mol Cell Cardiol $75,122-130$

47. Cenini G and Voos W (2016) Role of Mitochondrial Protein Quality Control in Oxidative Stress-induced Neurodegenerative Diseases. Curr Alzheimer Res 13, 164-173

48. Bragoszewski P, Turek M and Chacinska A (2017) Control of mitochondrial biogenesis and function by the ubiquitin - proteasome system. Open Biol 7, 17007

49. Suliman HB and Piantadosi CA (2016) Mitochondrial Quality Control as a Therapeutic Target. Pharmacol Rev 68, 20-48

50. Meyer A, Laverny G, Bernardi L et al (2018) Mitochondria: An Organelle of Bacterial Origin Controlling Inflammation. Front Immunol 9, 536

51. Archibald JM (2015) Endosymbiosis and Eukaryotic Cell Evolution. Curr Biol 25, R911-921

52. Barbalat R, Ewald SE, Mouchess ML and Barton GM (2011) Nucleic acid recognition by the innate immune system. Annu Rev Immunol 29, 185-214

53. Contis A, Mitrovic S, Lavie J et al (2017) Neutrophil-derived mitochondrial DNA promotes receptor activator of nuclear factor kappaB and its ligand signalling in rheumatoid arthritis. Rheumatology 56, 1200-1205

54. Shimada K, Crother TR, Karlin J et al (2012) Oxidized mitochondrial DNA activates the NLRP3 inflammasome during apoptosis. Immunity 36, 401-414

55. Bai J and Liu F (2019) The cGAS-cGAMP-STING Pathway: A Molecular Link Between Immunity and Metabolism. Diabetes 68, 1099-1108

56. Dorward DA, Lucas CD, Chapman GB, Haslett C, Dhaliwal K and Rossi AG (2015) The role of formylated peptides and formyl peptide receptor 1 in governing neutrophil function during acute inflammation. Am J Pathol 185, 1172-1184

57. Dahlgren C, Gabl M, Holdfeldt A, Winther $M$ and Forsman H (2016) Basic characteristics of the neutrophil receptors that recognize formylated peptides, a dangerassociated molecular pattern generated by bacteria and mitochondria. Biochem Pharmacol 114, 22-39

58. Raoof M, Zhang Q, Itagaki K and Hauser CJ (2010) Mitochondrial peptides are potent immune activators that activate human neutrophils via FPR-1. J Trauma 68, 1328-1332; discussion 1332-1324

59. Pan ZK, Chen LY, Cochrane CG and Zuraw BL (2000) fMet-Leu-Phe stimulates proinflammatory cytokine gene expression in human peripheral blood monocytes: the role of phosphatidylinositol 3-kinase. J Immunol 164, 404-411

60. Banoth B and Cassel SL (2018) Mitochondria in innate immune signaling. Transl Res 202, 52-68

61. Iyer SS, He Q, Janczy JR et al (2013) Mitochondrial Cardiolipin Is Required for Nlrp3 Inflammasome Activation. Immunity 39, 311-323

62. Chu CT, Bayir H and Kagan VE (2014) LC3 binds 
externalized cardiolipin on injured mitochondria to signal mitophagy in neurons Implications for Parkinson disease. Autophagy 10, 376-378

63. Allard B, Longhi MS, Robson SC and Stagg J (2017) The ectonucleotidases CD39 and CD73: Novel checkpoint inhibitor targets. Immunol Rev 276, 121-144

64. Amores-Iniesta J, Barbera-Cremades M, Martinez CM et al (2017) Extracellular ATP Activates the NLRP3 Inflammasome and Is an Early Danger Signal of Skin Allograft Rejection. Cell Rep 21, 3414-3426

65. Cauwels A, Rogge E, Vandendriessche B, Shiva $S$ and Brouckaert P (2014) Extracellular ATP drives systemic inflammation, tissue damage and mortality. Cell Death Dis 5, e1102-e1102

66. Eleftheriadis T, Pissas G, Liakopoulos V and Stefanidis I (2016) Cytochrome c as a Potentially Clinical Useful Marker of Mitochondrial and Cellular Damage. Front Immunol 7, 279

67. Lin ML, Zhan Y, Projetto Al et al (2008) Selective suicide of cross-presenting CD8(+) dendritic cells by cytochrome c injection shows functional heterogeneity within this subset. Proc Natl Acad Sci U S A 105, 3029-3034

68. Codina R, Vanasse A, Kelekar A, Vezys V and Jemmerson R (2010) Cytochrome c-induced lymphocyte death from the outside in: inhibition by serum leucine-rich alpha-2-glycoprotein-1. Apoptosis 15, 139-152

69. Pullerits $R$, Bokarewa $M$, Jonsson IM, Verdrengh $M$ and Tarkowski A (2005) Extracellular cytochrome c, a mitochondrial apoptosis-related protein, induces arthritis. Rheumatology 44, 32-39

70. Mittal M, Siddiqui MR, Tran K, Reddy SP and Malik AB (2014) Reactive Oxygen Species in Inflammation and Tissue Injury. Antioxid Redox Sign 20, 1126-1167

71. Kozlov AV, Lancaster JR, Meszaros AT and Weidinger A (2017) Mitochondria-meditated pathways of organ failure upon inflammation. Redox Biol 13, 170-181

72. Naik E and Dixit VM (2011) Mitochondrial reactive oxygen species drive proinflammatory cytokine production. J Exp Med 208, 417-420

73. Nakahira K, Haspel JA, Rathinam VAK et al (2011) Autophagy proteins regulate innate immune responses by inhibiting the release of mitochondrial DNA mediated by the NALP3 inflammasome. Nat Immunol $12,222-230$

74. West AP, Khoury-Hanold W, Staron M et al (2015) Mitochondrial DNA stress primes the antiviral innate immune response. Nature 520, 553-557

75. Tian J, Avalos AM, Mao SY et al (2007) Toll-like receptor 9-dependent activation by DNA-containing immune complexes is mediated by HMGB1 and RAGE. Nat Immunol 8, 487-496

76. Julian MW, Shao GH, Bao SY et al (2012) Mitochondrial Transcription Factor A Serves as a Danger Signal by Augmenting Plasmacytoid Dendritic Cell Responses to DNA. J Immunol 189, 433-443

77. Jacobs JL and Coyne CB (2013) Mechanisms of MAVS Regulation at the Mitochondrial Membrane. J Mol Biol 425, 5009-5019

78. Seth RB, Sun LJ, Ea CK and Chen ZJJ (2005) Identification and characterization of MAVS, a mitochondrial antiviral signaling protein that activates NF-kappa B and IRF3. Cell 122, 669-682

79. Subramanian N, Natarajan K, Clatworthy MR, Wang Z and Germain RN (2013) The Adaptor MAVS Promotes NLRP3 Mitochondrial Localization and Inflammasome Activation. Cell 153, 348-361

80. Castanier C, Garcin D, Vazquez A and Arnoult D (2010) Mitochondrial dynamics regulate the RIG--like receptor antiviral pathway. EMBO Rep 11, 133-138

81. Yasukawa K, Oshiumi H, Takeda M et al (2009) Mitofusin 2 Inhibits Mitochondrial Antiviral Signaling. Sci Signal 2, ra47

82. Tang ED and Wang CY (2009) MAVS Self-Association Mediates Antiviral Innate Immune Signaling. J Virol 83, 3420-3428

83. Vogel RO, Janssen RJRJ, van den Brand MAM et al (2007) Cytosolic signaling protein Ecsit also localizes to mitochondria where it interacts with chaperone NDUFAF1 and functions in complex I assembly. Gene Dev 21, 615-624

84. Geng J, Sun XF, Wang P et al (2015) Kinases Mst1 and Mst2 positively regulate phagocytic induction of reactive oxygen species and bactericidal activity. Nat Immunol $16,1142-1152$

85. Carneiro FRG, Lepelley A, Seeley JJ, Hayden MS and Ghosh S (2018) An Essential Role for ECSIT in Mitochondrial Complex I Assembly and Mitophagy in Macrophages. Cell Rep 22, 2654-2666

86. Shi HX, Liu X, Wang Q et al (2011) Mitochondrial Ubiquitin Ligase MARCH5 Promotes TLR7 Signaling by Attenuating TANK Action. PLoS Pathog 7, e1002057

87. Wilkins HM, Carl SM, Greenlief ACS, Festoff BW and Swerdlow RH (2014) Bioenergetic Dysfunction and Inflammation in Alzheimer's Disease: A Possible Connection. Front Aging Neurosci 6, 311

88. Wilkins HM, Weidling IW, Ji Y and Swerdlow RH (2017) Mitochondria-Derived Damage-Associated Molecular Patterns in Neurodegeneration. Front Immunol 8, 508

89. Bajwa E, Pointer CB and Klegeris A (2019) The Role of Mitochondrial Damage-Associated Molecular Patterns in Chronic Neuroinflammation. Mediators Inflammation 2019, 4050796

90. Wilkins HM, Koppel SJ, Weidling IW et al (2016) Extracellular Mitochondria and Mitochondrial Components Act as Damage-Associated Molecular Pattern Molecules in the Mouse Brain. J Neuroimmune Pharmacol 11, $622-628$

91. Guerreiro R, Wojtas A, Bras J et al (2013) TREM2 variants in Alzheimer's disease. $N$ Engl J Med 368 $117-127$

92. Korvatska O, Leverenz JB, Jayadev S et al (2015) R47H Variant of TREM2 Associated With Alzheimer Disease in a Large Late-Onset Family: Clinical, Genetic, and Neuropathological Study. JAMA Neurol 72, 920-927

93. Wang Y, Cella M, Mallinson K et al (2015) TREM2 lipid sensing sustains the microglial response in an Alzheimer's disease model. Cell 160, 1061-1071

94. Turnbull IR, Gilfillan S, Cella M et al (2006) Cutting edge: TREM-2 attenuates macrophage activation. J Immunol 177, 3520-3524 
95. Jiang T, Zhang YD, Chen Q et al (2016) TREM2 modifies microglial phenotype and provides neuroprotection in P301S tau transgenic mice. Neuropharmacology 105, 196-206

96. Podlesniy P, Figueiro-Silva J, Llado A et al (2013) Low cerebrospinal fluid concentration of mitochondrial DNA in preclinical Alzheimer disease. Ann Neurol 74, 655-668

97. Thubron EB, Rosa HS, Hodges A et al (2019) Regional mitochondrial DNA and cell-type changes in post-mortem brains of non-diabetic Alzheimer's disease are not present in diabetic Alzheimer's disease. Sci Rep 9, 11386

98. Ruggiero FM, Cafagna F, Petruzzella V, Gadaleta MN and Quagliariello E (1992) Lipid composition in synaptic and nonsynaptic mitochondria from rat brains and effect of aging. J Neurochem 59, 487-491

99. Pointer CB and Klegeris A (2017) Cardiolipin in Central Nervous System Physiology and Pathology. Cell Mol Neurobiol 37, 1161-1172

100. Petrosillo G, Matera M, Casanova G, Ruggiero FM and Paradies G (2008) Mitochondrial dysfunction in rat brain with aging Involvement of complex I, reactive oxygen species and cardiolipin. Neurochem Int 53, 126-131

101. Perier C, Tieu K, Guegan C et al (2005) Complex I deficiency primes Bax-dependent neuronal apoptosis through mitochondrial oxidative damage. Proc Natl Acad Sci U S A 102, 19126-19131

102. Little JP, Simtchouk S, Schindler SM et al (2014) Mitochondrial transcription factor A (Tfam) is a proinflammatory extracellular signaling molecule recognized by brain microglia. Mol Cell Neurosci 60, 88-96

103. Schindler SM, Frank MG, Annis JL, Maier SF and Klegeris A (2018) Pattern recognition receptors mediate pro-inflammatory effects of extracellular mitochondrial transcription factor A (TFAM). Mol Cell Neurosci 89, 71-79

104. Julian MW, Shao G, Vangundy ZC, Papenfuss TL and Crouser ED (2013) Mitochondrial transcription factor A, an endogenous danger signal, promotes TNF $\alpha$ release via RAGE- and TLR9-responsive plasmacytoid dendritic cells. PLoS One 8, e72354-e72354

105. Verdier Y, Zarandi M and Penke B (2004) Amyloid beta-peptide interactions with neuronal and glial cell plasma membrane: binding sites and implications for Alzheimer's disease. J Pept Sci 10, 229-248

106. Xie J, Mendez JD, Mendez-Valenzuela $\mathrm{V}$ and Aguilar-Hernandez MM (2013) Cellular signalling of the receptor for advanced glycation end products (RAGE). Cell Signal 25, 2185-2197

107. Lue LF, Walker DG, Brachova L et al (2001) Involvement of microglial receptor for advanced glycation endproducts (RAGE) in Alzheimer's disease: identification of a cellular activation mechanism. Exp Neurol 171, 29-45

108. Papaliagkas V, Anogeianakis G, Tsolaki M, Koliakos G and Kimiskidis V (2009) Prediction of Conversion from Mild Cognitive Impairment to Alzheimer's Disease by CSF Cytochrome C Levels and N200 Latency. Curr Alzheimer Res 6, 279-284

109. Takuma K, Yan SS, Stern DM and Yamada K (2005) Mitochondrial dysfunction, endoplasmic reticulum stress, and apoptosis in Alzheimer's disease. J Pharmacol Sci 97, 312-316

110. Krysko DV, Agostinis P, Krysko O et al (2011) Emerging role of damage-associated molecular patterns derived from mitochondria in inflammation. Trends Immunol 32, 157-164

111. Gouveia A, Bajwa E and Klegeris A (2017) Extracellular cytochrome $\mathrm{c}$ as an intercellular signaling molecule regulating microglial functions. Biochim Biophys Acta Gen Subj 1861, 2274-2281

112. Oyewole AO and Birch-Machin MA (2015) Mitochondriatargeted antioxidants. FASEB J 29, 4766-4771

113. Jauslin ML, Meier T, Smith RA and Murphy MP (2003) Mitochondria-targeted antioxidants protect Friedreich Ataxia fibroblasts from endogenous oxidative stress more effectively than untargeted antioxidants. FASEB J 17, 1972-1974

114. Gioscia-Ryan RA, LaRocca TJ, Sindler AL, Zigler MC, Murphy MP and Seals DR (2014) Mitochondria-targeted antioxidant (MitoQ) ameliorates age-related arterial endothelial dysfunction in mice. J Physiol 592, 2549-2561

115. Jin H, Kanthasamy A, Ghosh A, Anantharam V, Kalyanaraman B and Kanthasamy AG (2014) Mitochondriatargeted antioxidants for treatment of Parkinson's disease: preclinical and clinical outcomes. Biochimica et biophysica acta 1842, 1282-1294

116. Dashdorj A, Jyothi KR, Lim S et al (2013) Mitochondriatargeted antioxidant MitoQ ameliorates experimental mouse colitis by suppressing NLRP3 inflammasomemediated inflammatory cytokines. BMC Med 11, 178

117. Asano T, Koike M, Sakata S et al (2015) Possible involvement of iron-induced oxidative insults in neurodegeneration. Neurosci Lett 588, 29-35

118. Mena NP, Urrutia PJ, Lourido F, Carrasco CM and Nunez MT (2015) Mitochondrial iron homeostasis and its dysfunctions in neurodegenerative disorders. Mitochondrion 21, 92-105

119. Thomsen MS, Andersen MV, Christoffersen PR, Jensen MD, Lichota J and Moos T (2015) Neurodegeneration with inflammation is accompanied by accumulation of iron and ferritin in microglia and neurons. Neurobiol Dis 81, 108-118

120. Smigrodzki RM and Khan SM (2005) Mitochondrial microheteroplasmy and a theory of aging and age-related disease. Rejuvenation Res 8, 172-198

121. Casoli T, Spazzafumo L, Di Stefano G and Conti F (2015) Role of diffuse low-level heteroplasmy of mitochondrial DNA in Alzheimer's disease neurodegeneration. Front Aging Neurosci 7, 142-142

122. Onyango IG (2018) Modulation of mitochondrial bioenergetics as a therapeutic strategy in Alzheimer's disease. Neural Regen Res 13, 19-25

123. Jo A, Ham S, Lee GH et al (2015) Efficient Mitochondrial Genome Editing by CRISPR/Cas9. Biomed Res Int 2015, 305716

124. Hashimoto M, Bacman SR, Peralta $S$ et al (2015) MitoTALEN: A General Approach to Reduce Mutant mtDNA Loads and Restore Oxidative Phosphorylation Function in Mitochondrial Diseases. Mol Ther 23, 1592-1599 
125. Zhong Y, Hu YJ, Chen B et al (2011) Mitochondrial transcription factor $A$ overexpression and base excision repair deficiency in the inner ear of rats with D-galactose-induced aging. FEBS J 278, 2500-2510

126. Hayashi $Y$, Yoshida M, Yamato M et al (2008) Reverse of age-dependent memory impairment and mitochondrial DNA damage in microglia by an overexpression of human mitochondrial transcription factor a in mice. J Neurosci 28, 8624-8634

127. Xu S, Zhong M, Zhang L et al (2009) Overexpression of Tfam protects mitochondria against beta-amyloidinduced oxidative damage in SH-SY5Y cells. FEBS J 276, 3800-3809

128. Oka S, Leon J, Sakumi K et al (2016) Human mitochondrial transcriptional factor $A$ breaks the mitochondria-mediated vicious cycle in Alzheimer's disease. Sci Rep 6, 37889

129. Heneka MT, Kummer MP, Stutz A et al (2013) NLRP3 is activated in Alzheimer's disease and contributes to pathology in APP/PS1 mice. Nature 493, 674-678

130. Daniels MJ, Rivers-Auty J, Schilling T et al (2016) Fenamate NSAIDs inhibit the NLRP3 inflammasome and protect against Alzheimer's disease in rodent models. Nat Commun 7, 12504

131. Dempsey C, Rubio Araiz A, Bryson KJ et al (2017) Inhibiting the NLRP3 inflammasome with MCC950 promotes non-phlogistic clearance of amyloid-beta and cognitive function in APP/PS1 mice. Brain Behav Immun 61, 306-316

132. Yin J, Zhao F, Chojnacki JE et al (2018) NLRP3 Inflammasome Inhibitor Ameliorates Amyloid Pathology in a Mouse Model of Alzheimer's Disease. Mol Neurobiol 55, 1977-1987

133. Yang $Y$, Wang $H$, Kouadir $M$, Song $H$ and Shi $F$ (2019) Recent advances in the mechanisms of NLRP3 inflammasome activation and its inhibitors. Cell Death Dis 10,128

134. Lautrup S, Lou G, Aman Y, Nilsen H, Tao J and Fang EF (2019) Microglial mitophagy mitigates neuroinflammation in Alzheimer's disease. Neurochem Int 129, 104469
135. Fang EF, Hou Y, Palikaras K et al (2019) Mitophagy inhibits amyloid- $\beta$ and tau pathology and reverses cognitive deficits in models of Alzheimer's disease. Nat Neurosci 22, 401-412

136. Lei Q, Tan J, Yi S, Wu N, Wang Y and Wu H (2018) Mitochonic acid 5 activates the MAPK-ERK-yap signaling pathways to protect mouse microglial BV-2 cells against TNFalpha-induced apoptosis via increased Bnip3-related mitophagy. Cell Mol Biol Lett 23, 14

137. Zhou R, Yazdi AS, Menu P and Tschopp J (2011) A role for mitochondria in NLRP3 inflammasome activation. Nature 469, 221-225

138. Jiang S, Nandy P, Wang $W$ et al (2018) Mfn2 ablation causes an oxidative stress response and eventual neuronal death in the hippocampus and cortex. Mol Neurodegener 13, 5

139. Park J, Choi H, Min JS et al (2013) Mitochondrial dynamics modulate the expression of pro-inflammatory mediators in microglial cells. J Neurochem 127, 221-232

140. Kim H, Lee JY, Park KJ, Kim W-H and Roh GS (2016) A mitochondrial division inhibitor, Mdivi-1, inhibits mitochondrial fragmentation and attenuates kainic acid-induced hippocampal cell death. BMC Neurosci 17,33

141. Joshi AU, Minhas PS, Liddelow SA et al (2019) Fragmented mitochondria released from microglia trigger A1 astrocytic response and propagate inflammatory neurodegeneration. Nat Neurosci 22, 1635-1648

142. Akhter F, Chen D, Yan SF and Yan SS (2017) Mitochondrial Perturbation in Alzheimer's Disease and Diabetes. Prog Mol Biol Transl Sci 146, 341-361

143. Barber GN (2014) STING-dependent cytosolic DNA sensing pathways. Trends Immunol 35, 88-93

144. Liang Q, Seo GJ, Choi YJ et al (2014) Crosstalk between the CGAS DNA sensor and Beclin-1 autophagy protein shapes innate antimicrobial immune responses. Cell Host Microbe 15, 228-238

145. Sliter DA, Martinez J, Hao L et al (2018) Parkin and PINK1 mitigate STING-induced inflammation. Nature 561, 258-262 